\title{
Diseño de una micro-red virtual fotovoltaica, aplicando monitoreo energético en tiempo real, y estrategias de cambio de conducta para el ahorro de energía
}

\section{Design of a virtual photovoltaic microgrid, applying real-time energy monitoring, and behavior change theories for energy saving}

RENTERÍA-MACEDO, Francisco Daniel $\dagger$, GARCÍA-GUERRERO, Santiago Martín, HAROFALCÓN, Nicolás y CORONADO-MENDOZA, Alberto*

Universidad de Guadalajara, Centro Universitario de Tonalá, Instituto de Energía Renovable, Tonalá, Jalisco, México

ID $1^{\text {er }}$ Autor: Francisco Daniel, Rentería-Macedo / ORC ID: 0000-0003-3190-7046, Researcher ID Thomson: E-99222019, CVU CONACYT ID: 970060

ID $1^{\text {er }}$ Coautor: Santiago Martín, García-Guerrero / ORC ID: 0000-0001-5127-8027, Researcher ID Thomson: F-18542019, CVU CONACYT ID: 970236

ID $2^{\text {do }}$ Coautor: Nicolás, Haro-Falcón / ORC ID: 0000-0002-7139-7733, Researcher ID Thomson: F-5842-2019, CVU CONACYT ID: 388519

ID $3^{\text {er }}$ Coautor: Alberto, Coronado-Mendoza / ORC ID: 0000-0002-6283-4911, Researcher ID Thomson: F-3483-2019,

CVU CONACYT ID: 98169

DOI: $10.35429 /$ JOIE.2019.10.3.5.17

Recibido 19 de Marzo, 2019; Aceptado 30 de Junio, 2019

\section{Resumen}

La reforma energética mexicana de 2013 posibilitó el desarrollo de nuevos modelos de gestión e infraestructura energética. Sin embargo, existe una creciente y desatendida evidencia de la relevancia del análisis del comportamiento humano en el consumo energético. Por ello, esta investigación integra, de forma interdisciplinaria, el diseño de una micro-red virtual y un mercado energético en el Centro Universitario de Tonalá, de la Universidad de Guadalajara, aplicando monitoreo energético en tiempo real y teorías para el cambio de la conducta. Primeramente, se propone el diseño de una micro-red virtual de 5 edificios, cada uno con una planta virtual de generación, dimensionada a partir de su demanda histórica y la generación de la planta fotovoltaica de 499 kWp, instalada en el Centro Universitario. Se cuenta con monitoreo en tiempo real, de consumo y generación, desde mayo de 2018. Fue a partir de estos datos, que se dimensionaron las plantas virtuales. Paralelamente, se aplica el Modelo Transteórico de las etapas del cambio y la Teoría de la Difusión de las Innovaciones, para diseñar una intervención orientada al cambio de hábitos de consumo energético en la comunidad universitaria y fundamentar la implementación de un mercado energético piloto.

Micro-red, Monitoreo energético, Cambio de conducta

\begin{abstract}
The Mexican energy reform of 2013 enabled the development of new management models and energy infrastructure. However, there is growing and unattended evidence of the weight of human behavior analysis in energy consumption. Therefore, this research integrates, with an interdisciplinary approach, the design of a virtual microgrid and an energy market in the Tonalá Campus of the University of Guadalajara, with real-time energy monitoring and behavior change theories. Firstly, the design of a virtual microgrid of 5 buildings, each with a virtual generation plant, is proposed. Each one dimensioned based on its historical demand and the generation of a 499-kWp photovoltaic plant, installed at the Campus. Both consumption and generation have real-time monitoring installed since May 2018. It was from this data that the virtual power plant for each building was dimensioned. Parallelly, the Transtheoretical Model of the stages of change and the Diffusion of Innovation Theory, are applied to design an intervention to modify energy consumption habits in the Campus community and set the foundations of an energy market pilot program.
\end{abstract}

Microgrid, Energy monitoring, Behavior change

Citación: RENTERÍA-MACEDO, Francisco Daniel, GARCÍA-GUERRERO, Santiago Martín, HARO-FALCÓN, Nicolás y CORONADO-MENDOZA, Alberto. Diseño de una micro-red virtual fotovoltaica, aplicando monitoreo energético en tiempo real, y estrategias de cambio de conducta para el ahorro de energía. Revista de Ingeniería Innovativa. 2019. 3-10: 5-17

*Correspondencia al Autor (Correo electrónico: alberto.coronado@ cutonala.udg.mx)

$\dagger$ Investigador contribuyendo como primer Autor. 


\section{Introducción}

La eficiencia y el ahorro energético en edificaciones se han vuelto temas relevantes para hacer frente a la crisis socioambiental que representa el cambio climático, provocado principalmente por la emisión antropogénica de gases de efecto invernadero (Allouhi et al., 2015). Sin embargo, la investigación relacionada al consumo energético ha tenido principalmente un enfoque tecnológico, a pesar de la creciente evidencia de la importancia de integrar el estudio del comportamiento humano (Sovacool, 2014). La demanda de energía es afectada tanto por las decisiones y comportamiento de los usuarios, como por el rendimiento técnico $(\mathrm{Li}$, Hong \& Yan, 2014). Existe un creciente número de estudios que argumentan a favor de integrar a las ciencias sociales en el campo de la eficiencia y el ahorro energético (Stern, Sovacool \& Dietz, 2016; Sovacool, 2014; McKenzie-Mohr, 2011).

En respuesta al llamado a esta integración, diversos estudios han investigado cómo la visualización en tiempo real del consumo energético afecta las actitudes y conductas de los ocupantes de un edificio (Timm \& Deal, 2016; Buchanan, Russo \& Anderson, 2014; He, Greenberg \& Huang, 2010; Kaiser, Hübner \& Bogner, 2005). Por otro lado, en el contexto mexicano, la reforma energética de 2013 posibilitó el desarrollo de nuevos modelos de gestión e infraestructura energética (AlpizarCastro \& Rodríguez-Monroy, 2016).

El presente trabajo tiene por objetivo aportar a esta línea de investigación a través del diseño de una micro-red virtual fotovoltaica, aplicando monitoreo en tiempo real y estrategias para la adopción de nuevas conductas que promuevan el ahorro energético en una comunidad universitaria; específicamente, en el Centro Universitario de Tonalá (CUT), de la Universidad de Guadalajara (UDG).

\section{Contextualización: el caso del Centro Universitario de Tonalá}

El CUT cuenta con monitoreo en tiempo real del consumo de electricidad de 5 edificios, así como de la generación eléctrica de una planta fotovoltaica con capacidad instalada de 499 kWp, ubicada dentro del Centro Universitario.
El periodo de estudio considerado en esta investigación abarca desde octubre de 2018 hasta mayo de 2019. Por otro lado, como parte del trabajo del grupo de Gestores de Energía del Centro Universitario, se tiene la meta institucional de reducir un $20 \%$ el consumo de cada edificio. El presente trabajo es la primera etapa del futuro diseño e implementación de un Mercado Energético en el CUT; proyecto a cargo del Instituto de Energías Renovables (IER), con el fin de involucrar a la comunidad universitaria en el ahorro de energía.

La construcción de la planta de generación fotovoltaica dentro del Centro Universitario, el IER, así como las políticas universitarias de eficiencia energética, forman parte del Plan Universitario Integral de Transición Energética (PUITE), de la UDG (Operadora Universitaria de Energía, 2017).

\section{Micro-redes virtuales como herramienta conceptual}

El concepto de micro-red virtual se asemeja al de Planta de Generación Virtual (PGV), que puede definirse como una representación flexible de recursos energéticos distribuidos (Saboori, Mohammadi \& Taghe, 2011). En el presente proyecto, se define a las micro-redes virtuales como una representación conceptual y simplificada de una micro-red eléctrica, a través de la división virtual de una planta de generación real y su redistribución en PGVs. En este proyecto, se secciona la planta de generación fotovoltaica con la que cuenta el CUT, con el propósito de integrarse como herramienta conceptual a estrategias de cambio de conducta para el ahorro de energía.

\section{Monitoreo energético y teorías del cambio de la conducta}

Los factores que definen el comportamiento proambiental son tan complejos que el analizarlos con únicamente un modelo o teoría no es suficiente (Kollmuss \& Agyeman, 2002). Algunas teorías dentro de la psicología del comportamiento humano, mayormente utilizadas en el sector de la salud (Nutbeam, Harris \& Wise, 2010), han sido ya aplicadas para explicar la conducta humana en relación al ahorro de energía. 
Como la Teoría del comportamiento planeado, el Modelo de valores, creencias y normas (Kaiser, Hübner \& Bogner, 2005), y el Modelo Transteórico, que considera las distintas etapas en el proceso del cambio de la conducta (He, Greenberg \& Huang, 2010). En este último modelo, se teoriza que los individuos transitan por distintas etapas para adoptar hábitos: 1) estar inconscientes o desinteresados, 2) considerar la posibilidad del cambio, 3) prepararse para el mismo, 4) tomar acción, y, finalmente, 5) mantener el comportamiento deseado a través del tiempo (Nutbeam, Harris \& Wise, 2010). Por otro lado, la Teoría de la Difusión de las Innovaciones ha sido ampliamente utilizada para estudiar la adopción de nuevos métodos y tecnologías en grupos de población (Völlink, Meertens \& Midden, 2002).

La transición energética ha sido teorizada como un proceso sociotécnico (Geels \& Schot, 2007). Diversos estudios resaltan la importancia de volver visible el consumo de energía (Timm \& Deal, 2016; Buchanan, Russo \& Anderson, 2014; He, Greenberg \& Huang, 2010); de rematerializarlo en términos económicos y ambientales (Burgess \& Nye, 2008). El incremento en las posibilidades de realizar una transición hacia conductas que aporten a la sustentabilidad debe basarse en intervenciones que busquen comprender el comportamiento humano y las barreras al cambio (McKenzieMohr, 2011). Motivar una transición de conducta hacia un consumo energético sustentable es un problema psicológico, social y culturalmente complejo (He, Greenberg \& Huang, 2010).

Por ejemplo, Timm \& Deal (2016) realizaron una investigación que tuvo lugar en 4 edificios de diferentes centros universitarios en Illinois, Estados Unidos, donde se logró que los usuarios adoptaran nuevos hábitos de ahorro energético al ser informados sobre el consumo de energía eléctrica mediante la proyección de datos de consumo en tiempo real en pantallas, acompañado de campañas de cambio de conducta. Como resultado, se ahorró entre 53$60 \%$ del consumo eléctrico. Esto reafirma lo que Sun et al. (2014) y Petersen et al. (2007) concluyen en su investigación; hasta el 30-50\% del consumo energético total de edificios está relacionado a la conducta de los ocupantes y a sus incentivos.
La siguiente sección detalla la metodología seguida para el monitoreo de generación y consumo de electricidad en el CUT, la asignación de paneles a las PGVs que conforman la micro-red virtual, el diseño de una encuesta basada en teorías para el cambio de la conducta y el diseño de estrategias para el ahorro de energía, aplicando teorías del cambio de la conducta. Consecutivamente, la sección 3 muestra los resultados obtenidos al seguir lo establecido en la sección 2. En la discusión se interpretan los resultados, se detallan sus similitudes con otros trabajos y sus principales limitaciones. Finalmente, las conclusiones hacen énfasis en las principales aportaciones de este trabajo y sugerencias para darle continuación.

\section{Metodología}

\section{Integración multidisciplinaria}

La Figura 1, por medio de un diagrama de Venn, muestra la integración de las 3 principales dimensiones de este proyecto: 1) monitoreo energético en tiempo real, 2) teorías del cambio de la conducta y 3 ) ahorro de energía.

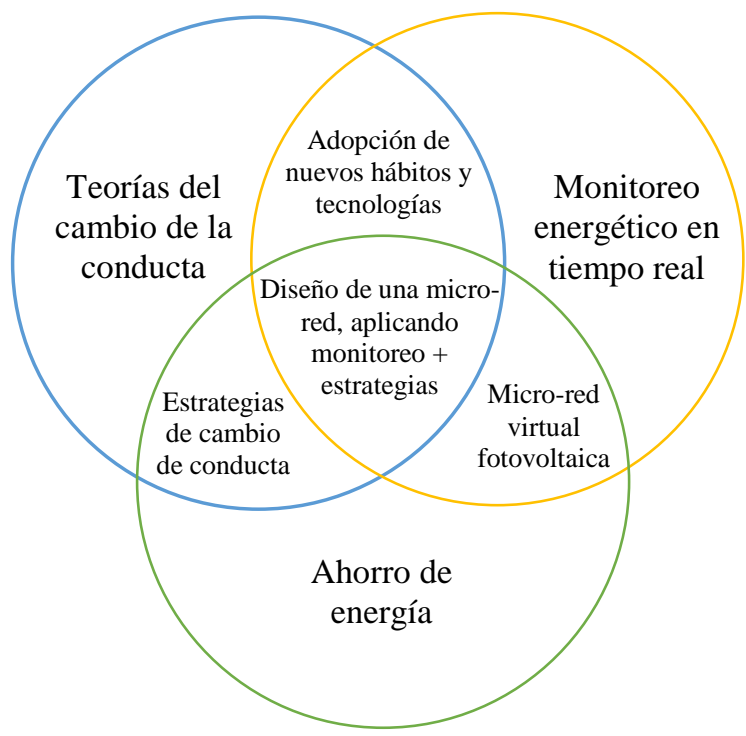

Figura 1 Integración de las dimensiones del diseño de una micro-red virtual integrando monitoreo en tiempo real y teorías para el cambio de conducta

Fuente: Elaboración Propia

Las intersecciones entre las 3 dimensiones en la Figura 1 muestran los subtemas que convergen para sustentar el diseño de una micro-red virtual fotovoltaica que integre monitoreo en tiempo real y teorías del cambio de la conducta para el ahorro de energía. 
Primero, la adopción de nuevos hábitos y tecnologías busca utilizar teorías del cambio de la conducta para facilitar la adopción de los monitores energéticos dentro de la comunidad universitaria. Segundo, dichas teorías también tienen el fin de ser aplicadas en el diseño de estrategias de cambio de conducta para el ahorro de energía. Finalmente, el concepto de micro-red fotovoltaica es utilizado como una herramienta conceptual para facilitar el ahorro de energía a través de monitoreo energético en tiempo real.

\section{Monitoreo en tiempo real de generación y consumo}

El monitoreo de generación y consumo de electricidad abarcó desde octubre de 2018 hasta mayo de 2019. Durante este periodo se registraron los datos de consumo de 5 edificios: biblioteca, edificio de salud, edificio de artesanías, comedor y el Instituto de Energía Renovable (IER); así como la generación en la planta fotovoltaica, con capacidad instalada de $499 \mathrm{kWp}$, proporcionada por 1560 paneles fotovoltaicos, conectados a 10 inversores. Los datos de generación fueron obtenidos a través de una plataforma digital de monitoreo, proporcionada por el fabricante de los inversores.

En cuanto al monitoreo del consumo, se calibraron 213 sensores de carga repartidos entre los 5 edificios contemplados en la propuesta de la micro-red. Cabe mencionar que el IER cuenta con monitoreo parcial. En futuras etapas del proyecto se planea extender el monitoreo a la totalidad del IER y a los demás edificios del CUT. Finalmente, la recolección de datos de consumo se dividió en tres periodos: 1) laboral con clases, 2) laboral sin clases y 3) vacacional, para así comparar el consumo entre ellos e identificar oportunidades de ahorro de energía.

\section{Propuesta de asignación de paneles}

Como política universitaria, se tiene el objetivo de reducir un $20 \%$ el consumo actual de cada edificio. La asignación de paneles fotovoltaicos para la PGV de cada edificio se basa en esta meta. A partir de los datos de generación y consumo meta por edificio, se procedió a dividir la planta de generación fotovoltaica en PGVs.
Primero, se calculó la generación diaria promedio por panel $\left(G_{p p}\right)$, que es simplemente la relación entre la sumatoria de la generación promedio diaria $(i)$ de cada uno de los 10 inversores $\left(n_{\text {inversores }}\right)$, representados por el subíndice $j$, y el número total de paneles ( $\left.n_{\text {paneles }}\right)$, como se representa en la Ecuación 1. Lo anterior con el fin de alcular los $\mathrm{kWh}$ que produce cada panel en promedio por día.

$G_{p p}=\frac{\sum_{j=1}^{n_{\text {inversores }}} i_{j}}{n_{\text {paneles }}}$

Donde:

$G_{p p}=$ generación promedio diaria por panel $\left(\frac{k W h}{\text { panel }}\right)$

$n_{\text {paneles }}=$ número de paneles

$n_{\text {inversores }}=$ número de inversores

$i_{j}=$ generación promedio diaria de cada inversor $j(\mathrm{kWh})$

Finalmente, como lo indica la Ecuación 2 , el consumo promedio diario de cada edificio $(C)$ es dividido entre la generación promedio diaria por panel $\left(G_{p p}\right)$, proporcionada por la Ecuación 1, para obtener la cantidad de paneles de cada PGV ( $\left.P G V_{\text {paneles }}\right)$. Para calcular el valor de consumo promedio diario de cada edificio, se consideró únicamente el periodo laboral con clases, ya que es cuando se tiene mayor consumo y la mayoría de los usuarios realiza actividades dentro del Centro Universitario. Al valor resultante de utilizar la Ecuación 2 se le aplica una función techo, que consiste en asignarle el número entero más próximo por exceso. Cabe mencionar que el análisis técnico de la micro-red propuesta no forma parte del alcance de este proyecto.

$P G V_{\text {paneles }}=\frac{C}{G_{p p}}$

Donde:

$G_{p p}=$ generación promedio diaria por panel $\left(\frac{k W h}{\text { panel }}\right)$

$\mathrm{C}=$ consumo promedio diario de cada edificio (kWh)

$P G V_{\text {paneles }}=$ paneles asignados a la $\mathrm{PGV}$ 


\section{Diseño de una encuesta, aplicando teorías para el cambio de la conducta}

La encuesta tuvo el objetivo de recabar información sobre los niveles de aceptación de varias estrategias de ahorro de energía en edificaciones para ser aplicadas en el CUT, así como sus niveles de conciencia, percepción de poder sobre su entorno e interés en cambiar. El diseño de la encuesta se basó en la Teoría de la Difusión de las Innovaciones y el Modelo Transteórico, ya que han sido aplicados en otros trabajos donde se ha estudiado cómo las tecnologías de retroalimentación energética pueden coadyuvar a técnicas y teorías existentes dentro de la psicología motivacional, para incentivar conductas de uso energético sustentable (He, Greenberg \& Huang, 2010; Völlink, Meertens \& Midden, 2002).

En este trabajo, la integración del Modelo Transteórico y la Teoría de la Difusión de las Innovaciones se utiliza para considerar las preferencias de cada segmento de la población y las etapas del cambio que deberán experimentar. Esto, debido a que se ha señalado en otras investigaciones que la información en tiempo real del consumo no impacta a la totalidad de la comunidad, como es el caso del estudio realizado por Timm \& Deal (2016), donde se demostró que no hubo cambios significativos de actitud en los estudiantes, en comparación con el personal administrativo y profesores.

Por consiguiente, la encuesta considera los roles de los usuarios, para su posterior uso en el diseño de las estrategias de cambio de la conducta. Esto da pauta para la clasificación de los encuestados y las tendencias en el interés de cambiar, ya que Timm \& Deal (2016) han concluido que un segmento importante de los usuarios puede no tener interés explícito en el ahorro de energía, especialmente en edificaciones sobre las que no perciben tener control. A continuación, se explica brevemente el Modelo Transteórico y la Teoría de la Difusión de las Innovaciones, que sirvieron de base para el diseño de la encuesta.

\section{Modelo Transteórico}

Este modelo explica las etapas que implica un cambio de conducta y establece que es más similar a un proceso que a un evento. Dicho modelo se divide en 5 etapas, cada una con un proceso de cambio necesario para avanzar a la siguiente fase (Nutbeam, Harris \& Wise, 2010). En la Tabla 1, se detalla cada una de las 5 categorías en las que se clasifican a los individuos, dependiendo de la etapa del proceso de cambio en la que se encuentren. En cada una de estas etapas de cambio se recomienda aplicar diferentes procesos para el individuo.

\section{Etapas de cambio}

Proceso de cambio

Pre-contemplación - esta etapa $\quad$ Concientización

describe a los individuos que no

consideran cambiar, y a aquellos

que no tienen la intención de

hacerlo.

Contemplación - individuos Reconocimiento de

que están interesados en los beneficios del

cambiar una conducta en cambio

específico.

Determinación - etapa en la Identificación de que un individuo está barreras

comprometido a hacer un cambio.

Acción - etapa en la que el

cambio de conducta ha iniciado.

Mantenimiento - etapa del

cambio sostenido, donde se

lograron los objetivos.

Tabla 1 Descripción de las etapas y proceso de cambio del Modelo Transteórico

Fuente: Nutbeam, Harris \& Wise, 2010

\section{Teoría de la Difusión de las Innovaciones}

Al igual que el Modelo Transteórico, la Teoría de la Difusión de las Innovaciones tiene 5 categorías en las que se clasifica a la población, pero en este caso, con base en la velocidad con la que una innovación es adoptada. La Tabla 2 indica el porcentaje y características de la población que corresponde a cada categoría. 


\begin{tabular}{|l|l|}
\hline \multicolumn{1}{|c|}{ Clasificación } & \multicolumn{1}{|c|}{ Segmento } \\
\hline Innovadores & $\begin{array}{l}\text { Población que adopta nuevas ideas } \\
\text { de manera rápida (2-3\%). }\end{array}$ \\
\hline $\begin{array}{l}\text { Adoptadores } \\
\text { tempranos }\end{array}$ & $\begin{array}{l}\text { Población que puede pertenecer a la } \\
\text { corriente mayoritaria de la } \\
\text { comunidad, pero con mayor } \\
\text { tendencia al cambio debido a } \\
\text { recursos propios (10-15\%). }\end{array}$ \\
\hline $\begin{array}{l}\text { Mayoría } \\
\text { temprana }\end{array}$ & $\begin{array}{l}\text { Población que tiende a cambiar } \\
\text { persuadidos por los beneficios del } \\
\text { cambio (30-35\%). }\end{array}$ \\
\hline Mayoría tardía & $\begin{array}{l}\text { Parte de la población que depende del } \\
\text { tiempo y el establecimiento claro de } \\
\text { los beneficios (30-35\%). }\end{array}$ \\
\hline Rezagados & $\begin{array}{l}\text { Población conservadora yegada a la } \\
\text { propuesta de un cambio (10-20\%). }\end{array}$ \\
\hline
\end{tabular}

Tabla 2 Clasificación general de los segmentos de una población, según la Teoría de la Difusión de las Innovaciones

Fuente: Nutbeam, Harris \& Wise, 2010

A partir de lo anterior, fue que se diseñó la encuesta que se aplicó a la comunidad universitaria del CUT. Se utilizó la escala de Likert para evaluar el deseo de aprender y cambiar, el nivel de conciencia, la percepción de poder sobre el entorno y las actitudes de los distintos segmentos de usuarios que utilizan los 5 edificios que conforman la micro-red. Como es propuesto por Timm \& Deal (2016), dentro de la encuesta se incluyeron imágenes de los edificios que están contemplados, para una comprensión clara.

Además, se aplicaron dos preguntas clave por cada edificio: 1) ¿Cuántas horas a la semana, en promedio, realiza actividades en este edificio? y 2) ¿Con qué frecuencia realiza acciones directas que impliquen un consumo energético?, para esta última pregunta se brinda un rango de actividades individuales que consumen energía.

Asimismo, se dieron tres opciones a evaluar para la visualización del consumo en tiempo real. Dichas opciones fueron: 1) orbes de energía con interfaz, 2) orbes de energía sin interfaz y 3) monitores de energía. En la Figura 2 , se muestra un orbe de energía sin interfaz. La visualización del consumo en tiempo real es mediante los colores verde, naranja y rojo, que indican si un edificio está por debajo de su consumo meta, si está cerca del límite o si lo ha sobrepasado, respectivamente.
Los orbes de energía con interfaz tienen el mismo funcionamiento, pero con visualización de tarifas. Timm \& Deal (2016) enfatizan el potencial del uso de orbes indicadores de consumo energético para volver visible la intensidad del consumo de electricidad en una edificación.

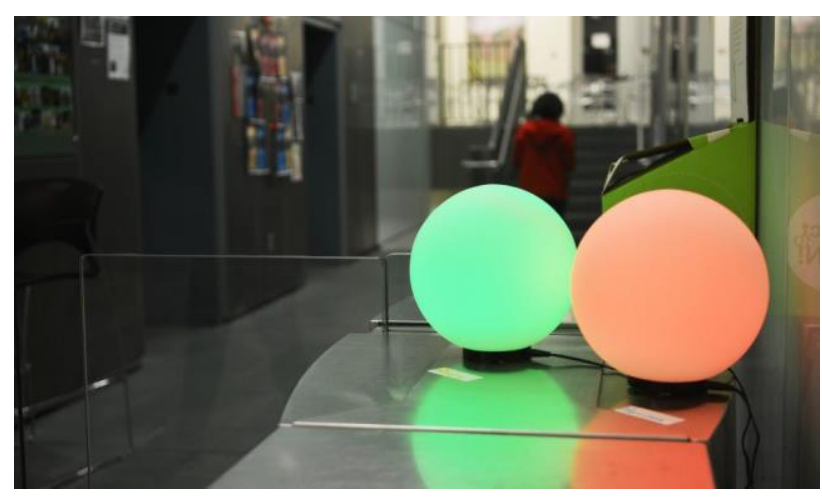

Figura 2 Orbe indicador de consumo energético Fuente: Block, 2014

La tercera propuesta es utilizar monitores (Figura 3). A diferencia de los orbes, en la interfaz del monitor se hace una comparación de los consumos actuales contra los previos, de manera mensual, semanal y diaria, con el propósito de indicar si hubo una tendencia de crecimiento o reducción, tanto de consumo como de generación, en dichos periodos.

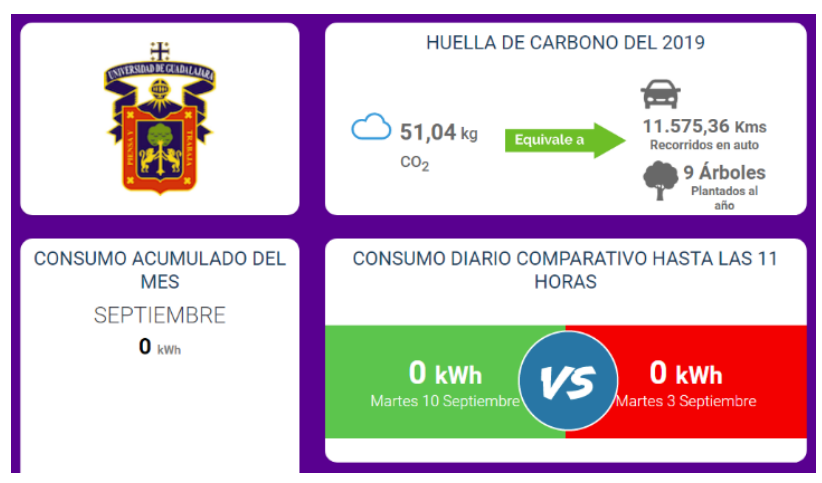

Figura 3 Propuesta de interfaz para monitor de generación y consumo energético en tiempo real

Fuente: Elaboración Propia

Además, en la encuesta se presentan estrategias para el cambio de conducta, divididas en dos grupos: estímulos y medidas formativas/correctivas. Primero, el objetivo es evaluar en qué medida sería aceptado cada estímulo para ser aplicado en el Centro Universitario, considerando que los usuarios de cierto edificio mantienen un uso moderado de la energía. Estos se enlistan a continuación: 1) becas, 2) equipo/mobiliario, 3) evento cultural, 4) intervenciones artísticas y 5) picnic. 
De forma similar, se proponen medidas correctivas para ser aplicadas en caso de que los usuarios de un edificio mantengan un uso excesivo de la energía. Estas son las siguientes: 1) asistencia a cursos de ahorro de energía, 2) participación en talleres de ahorro de energía, 3) programa de servicio social: cuidado de áreas verdes y forestación y 4) programa de servicio social: limpieza de paneles. La evaluación de estas estrategias se hace también mediante la escala de Likert. La encuesta fue distribuida por medio de correo electrónico, a través de las autoridades del CUT.

\section{Diseño de estrategias para la adopción de hábitos de ahorro energético}

De acuerdo con los resultados de la encuesta, las estrategias se clasifican por segmentos de población, de acuerdo con la etapa de cambio correspondiente y facilidad para adoptar innovaciones, dependiendo de los niveles de conciencia, actitudes y voluntad para cambiar, mostrados por los encuestados, para así contar con un rango de medidas que cubran la totalidad del proceso del cambio de la conducta. El diseño de estrategias se basa en las conclusiones de estudios similares y los resultados de la encuesta descrita en la sección anterior.

La asignación de estrategias consideró que además de implementar dispositivos que indiquen el consumo en tiempo real, es recomendado acompañarlo de una conceptualización con enfoque ambiental o de salud, a diferencia de uno monetario, ya que se ha concluido en otros estudios que esto brinda resultados sostenidos a largo plazo (Asensio \& Delmas, 2016).

De forma paralela, se considera que es necesario aplicar políticas de ahorro energético orientadas al aprendizaje, ya que se ha demostrado que pueden ser más efectivas que el monitoreo por sí mismo (Asensio \& Delmas, 2016). Conjuntamente, se ha reportado que la información en tiempo real no incita el completo interés de una comunidad, así que es importante complementarlo con otras medidas (Lynham et al., 2016; Timm \& Deal, 2016; He, Greenberg \& Huang, 2010). Por esta razón, la asignación de estrategias es individual, pero integrada a cada diferente segmento de la población y etapa de cambio.

\section{Resultados}

Esta sección presenta los resultados de seguir la metodología descrita anteriormente. Se desarrollan los datos del monitoreo en tiempo real y la subsecuente asignación de paneles basada en estos datos. Posteriormente, se presentan los puntos más relevantes de la encuesta, y el diseño posterior de estrategias integrando dichos hallazgos con el Modelo Transteórico y la Teoría de la Difusión de las Innovaciones.

\section{Monitoreo en tiempo real de generación y consumo}

En el Gráfico 1, se muestran los datos de monitoreo de cada uno de los 10 inversores de la planta de generación fotovoltaica. A partir de estos, se calculó un promedio de generación diaria por panel, utilizando la Ecuación 1. Se observa que, con excepción del inversor 2 , los demás tienen una generación promedio diaria mayor a los $170 \mathrm{kWh}$.

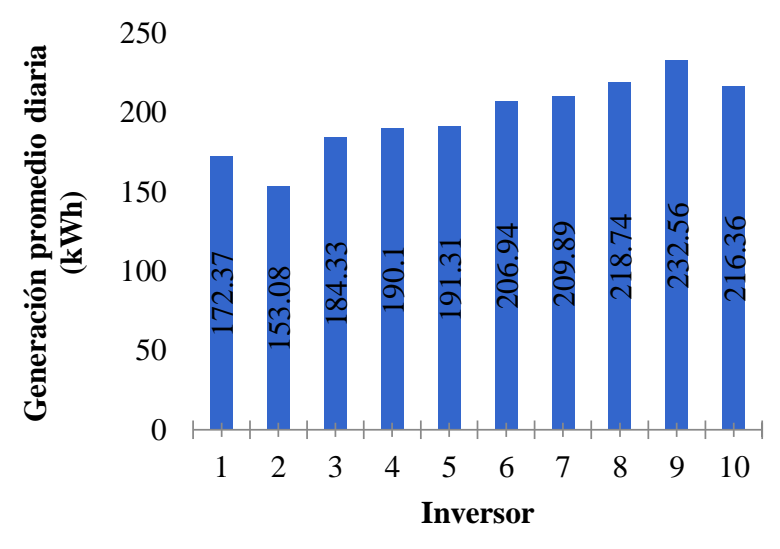

Gráfico 1 Generación diaria promedio por inversor Fuente: Elaboración Propia

Por otro lado, el consumo de los 5 edificios es presentado en el Gráfico 2. En este, se observa el consumo total promedio por mes, de octubre de 2018 a mayo de 2019 , de los 5 edificios, siendo la biblioteca el de mayor consumo, y el IER el de menor, debido al monitoreo parcial de este último. Después de octubre, se ve reflejada una reducción del consumo, para incrementar gradualmente después de enero, comportamiento posiblemente relacionado a las fluctuaciones estacionales de temperatura. 


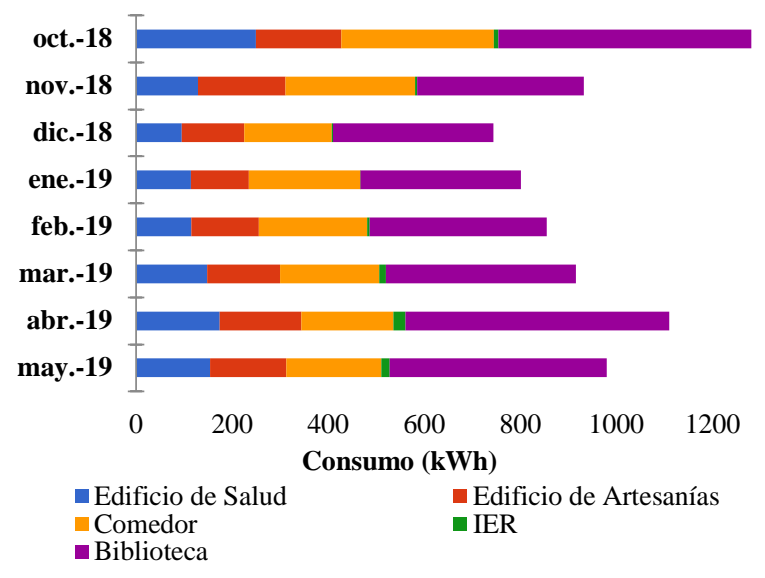

Gráfico 2 Consumo promedio mensual por edificio en periodo laboral con clases

Fuente: Elaboración Propia

El Gráfico 3 compara el consumo por edificio entre tres periodos: 1) laboral con clases, 2) laboral sin clases y 3) vacacional. Como se puede observar, en todos los edificios hubo mayor consumo energético en los días laborales con clases en comparación con los otros periodos. Sin embargo, el comedor presenta una situación diferente, ya que el consumo aumentó durante el periodo laboral sin clases. Este aumento podría deberse a la necesidad de refrigerar alimentos incluso en tiempo de menor actividad.

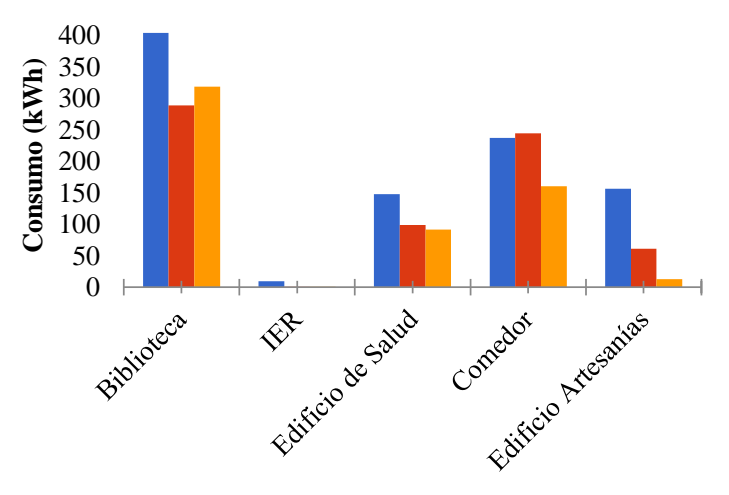

— Dias laborales $\quad$ Dias laborales sin clases $\square$ Periodo vacacional

Gráfico 3 Comparación del consumo por edificio en distintos periodos

Fuente: Elaboración propia

\section{Propuesta de asignación de paneles}

Aplicando la Ecuación 1, descrita en la metodología, la generación diaria promedio por panel es de $1.26 \mathrm{kWh}$. Como se mencionó anteriormente, este valor resulta de la relación entre la cantidad total de paneles que hay en la planta de generación fotovoltaica (1560) y la sumatoria de la generación promedio diaria de cada uno de los 10 inversores.
También, se tomó en cuenta la reducción del $20 \%$ del consumo total de cada edificio. Posteriormente, utilizando la Ecuación 2, que relaciona el valor de generación diaria por panel y el consumo meta, se asignaron los paneles correspondientes a la PGV de cada edificio. En la Tabla 3, se presenta el consumo meta por edificio, los paneles asignados a cada PGV y su porcentaje en relación con el total de paneles. En esta, se observa que el ahorro de paneles utilizados con la propuesta del consumo meta es de 152 , equivalente a casi el $10 \%$ de la planta fotovoltaica. Dicha asignación dejaría aproximadamente al $60 \%$ de los paneles disponibles para ser asignados a otros edificios en futuras etapas del proyecto. La propuesta de asignación de paneles por edificio es presentada en el Gráfico 4.

\begin{tabular}{|l|r|r|r|}
\hline Edificio & $\begin{array}{c}\text { Consumo } \\
\text { meta diario } \\
\text { (kWh) }\end{array}$ & $\begin{array}{c}\text { Paneles } \\
\text { asignados }\end{array}$ & $\begin{array}{c}\text { Porcentaje } \\
\text { de paneles }\end{array}$ \\
\hline Biblioteca & 323.472 & 256 & $16.4 \%$ \\
\hline IER & 7.286 & 6 & $0.4 \%$ \\
\hline $\begin{array}{l}\text { Edificio de } \\
\text { Salud }\end{array}$ & 118.257 & 94 & $6.0 \%$ \\
\hline Comedor & 189.754 & 150 & $9.6 \%$ \\
\hline $\begin{array}{l}\text { Edificio de } \\
\text { Artesanías }\end{array}$ & 125.325 & 99 & $6.3 \%$ \\
\hline Ahorro & $\mathbf{1 9 1 . 0 2 4}$ & $\mathbf{1 5 2}$ & $\mathbf{9 . 7 \%}$ \\
\hline Restante & $\mathbf{1 0 2 0 . 6 2 0}$ & $\mathbf{8 0 3}$ & $\mathbf{5 1 . 5 \%}$ \\
\hline
\end{tabular}

Tab1a 3 Propuesta de asignación de paneles por edificio en función de su consumo

Fuente: Elaboración Propia

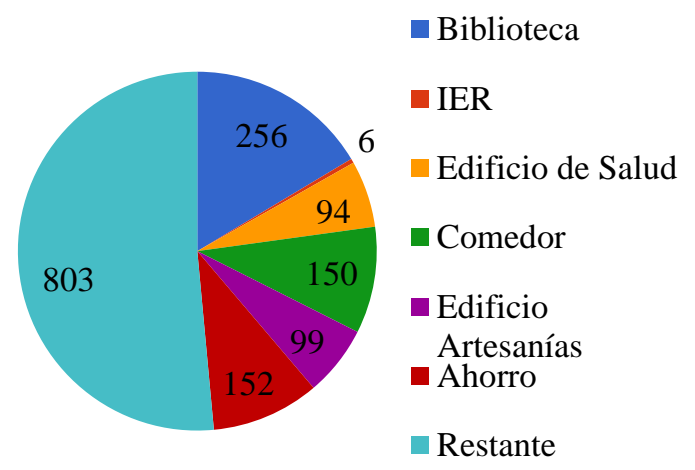

Gráfico 4 Asignación de paneles para las PGVs por edificio

Fuente: Elaboración Propia

\section{Resultados de la encuesta, aplicando teorías para el cambio de la conducta}

La encuesta fue contestada por un total de 53 miembros de la comunidad universitaria del CUT. El Gráfico 5 presenta las tendencias de aceptación por parte de la comunidad universitaria, en cuanto a los estímulos contemplados en el diseño de estrategias.

RENTERÍA-MACEDO, Francisco Daniel, GARCÍA-GUERRERO, Santiago Martín, HARO-FALCÓN, Nicolás y CORONADO-MENDOZA, Alberto. Diseño de una micro-red virtual fotovoltaica, aplicando monitoreo energético en tiempo real, y estrategias de cambio de conducta para el ahorro de energía. Revista de Ingeniería Innovativa. 2019. 
La mayoría señaló estar de acuerdo con todos los estímulos propuestos. Sin embargo, fueron las becas, con $65.4 \%$, y el equipo para los edificios, con $67.4 \%$, en donde se obtuvo mayor aceptación. Cabe mencionar que la suma de los segmentos de algunas categorías de las gráficas puede no sumar el $100 \%$ debido al redondeo aplicado.

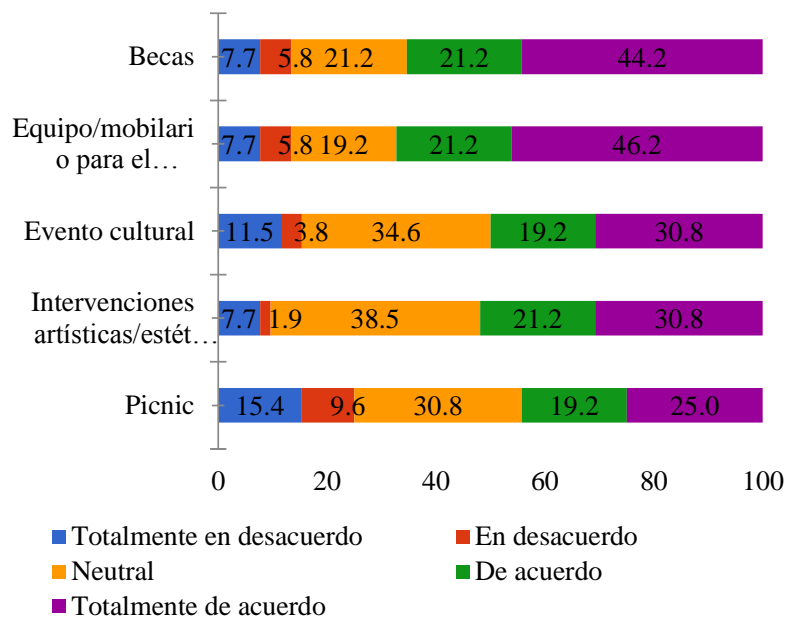

Gráfico 5 Percepción de propuestas de estímulos para el ahorro de energía

Fuente: Elaboración Propia

Respecto a las medidas formativas/correctivas propuestas para el diseño de estrategias de ahorro de energía, en el Gráfico 6 se presenta la aceptación de dichas medidas. Se aprecia que la mayoría de los encuestados está de acuerdo con la implementación de cada una de estas. La ejecución de programas de servicio social, como el cuidado de áreas verdes y forestación, obtuvo el 73.6\%, siendo la estrategia de mayor aceptación para ser implementada como medida correctiva para los usuarios de los edificios que tengan un uso excesivo de energía eléctrica.

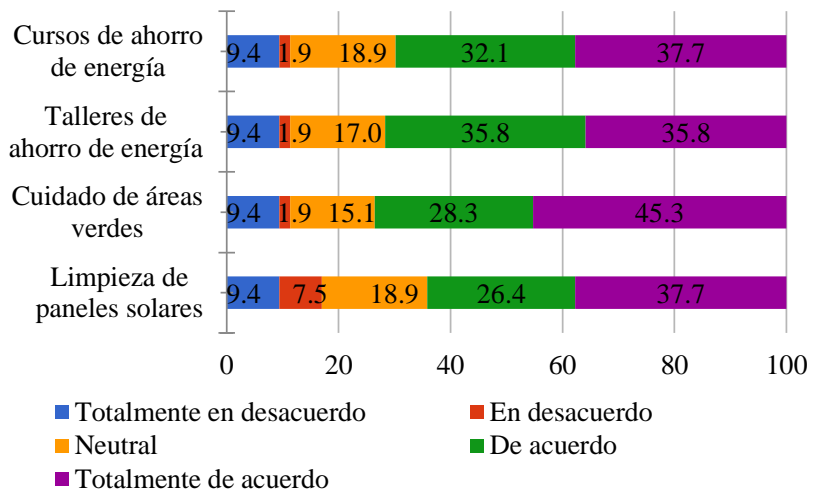

Gráfico 6 Percepción de propuestas de medidas correctivas para el ahorro de energía

Fuente: Elaboración Propia
El Gráfico 7 compara la aprobación de las políticas de estímulos y las políticas correctivas por parte de los encuestados. La mayoría de la población prefiere la aplicación de medidas correctivas.

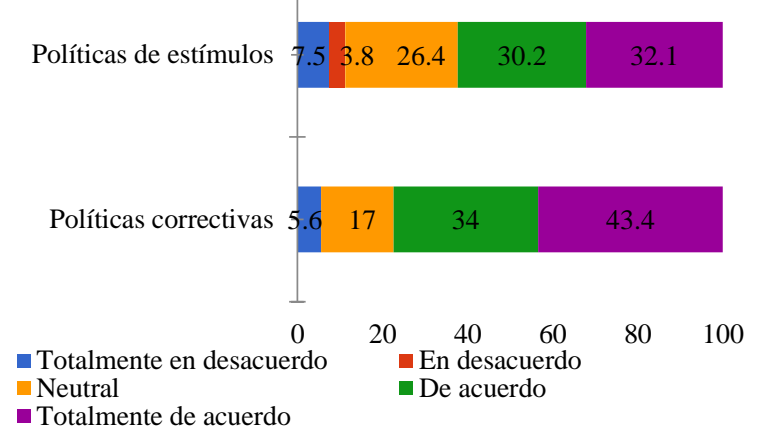

Gráfico 7 Comparativa de aceptación entre políticas de estímulos y correctivas

Fuente: Elaboración Propia

Para una mejor comprensión de las percepciones y actitudes de los encuestados, se analizaron 7 categorías que evalúan los grados de conciencia, actitud y percepción de capacidad de la población. En el Gráfico 8, se presenta cada una de las categorías que se tomaron en cuenta para la evaluación de los encuestados. De manera general, se observaron actitudes proambientales, aunque es necesario implementar estrategias que se enfoquen principalmente en el impulso del interés de cambio, acción y percepción de poder de la comunidad universitaria.

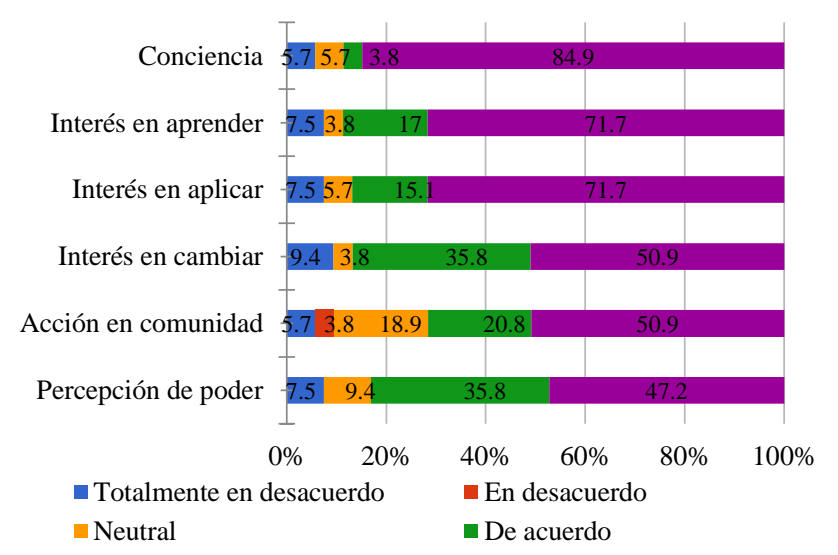

Gráfico 8 Percepción de conciencia, actitud y capacidad Fuente: Elaboración Propia

Dentro del diseño de la encuesta, se propusieron tres opciones a evaluar para la visualización del consumo en tiempo real, cuyo funcionamiento fue detallado en la metodología. En el Gráfico 9 se muestra de forma general que la mayor parte de los encuestados está de acuerdo con la implementación de tecnologías que vuelvan visible el consumo de los edificios.

RENTERÍA-MACEDO, Francisco Daniel, GARCÍA-GUERRERO, Santiago Martín, HARO-FALCÓN, Nicolás y CORONADO-MENDOZA, Alberto. Diseño de una micro-red virtual fotovoltaica, aplicando monitoreo energético en tiempo real, y estrategias de cambio de conducta para el ahorro de energía. Revista de Ingeniería Innovativa. 2019. 
$\mathrm{Si}$ bien las tres opciones mostraron niveles de aprobación similares, se observó que encuestados prefieren los orbes de energía, particularmente los que no cuentan con interfaz.

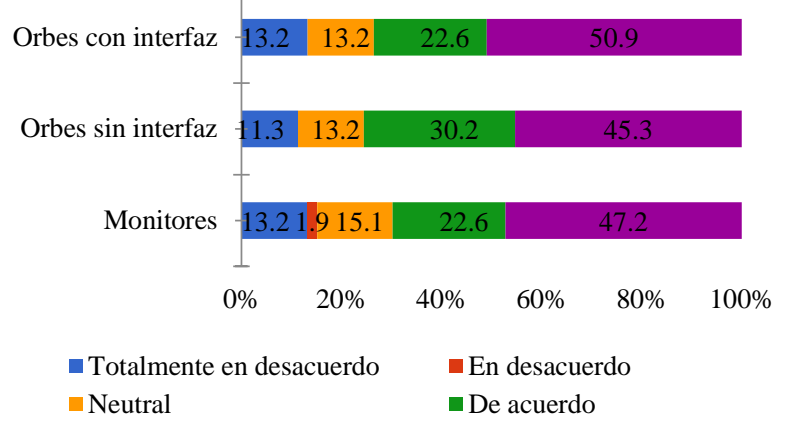

Gráfico 9 Aceptación de métodos de visualización del consumo en tiempo real

Fuente: Elaboración Propia

\section{Estrategias propuestas}

Este apartado relaciona los estímulos y las medidas formativas/correctivas, con las etapas del cambio del Modelo Transteórico y los segmentos de población de la Teoría de la Difusión de las Innovaciones, con base en los resultados de la encuesta y las conclusiones de estudios similares, mencionados en la metodología. En la Tabla 4, se muestran las estrategias que serán implementadas considerando las etapas del cambio del Modelo Transteórico. Particularmente, la propuesta de la creación de un sitio web, y sus distintas funciones, se origina de las observaciones de $\mathrm{He}$, Greenberg \& Huang (2010). El programa de mentoría proviene de las sugerencias proporcionadas por los encuestados.

\begin{tabular}{|l|l|}
\hline \multicolumn{2}{|c|}{$\begin{array}{l}\text { Etapas de } \\
\text { cambio }\end{array}$} \\
\hline $\begin{array}{l}\text { Pre- } \\
\text { contemplación }\end{array}$ & $\begin{array}{l}\text { Publicar pros y contras, en términos } \\
\text { monetarios y de emisiones, de un } \\
\text { comportamiento no sustentable, apelando a } \\
\text { normas sociales - sitio web y redes } \\
\text { sociales. } \\
\text { Consejos de ahorro - Monitores. } \\
\text { Orbes de energía. }\end{array}$ \\
\hline Contemplación & $\begin{array}{l}\text { Asistencia a cursos de ahorro de energía. } \\
\text { Historias de estilos de vida sustentable - } \\
\text { sitio web y redes sociales. } \\
\text { Orbes de energía. }\end{array}$ \\
\hline Determinación & $\begin{array}{l}\text { Participación en talleres de ahorro de } \\
\text { energía. } \\
\text { Conexión con mentores en etapas de acción } \\
\text { y mantenimiento - sitio web y redes } \\
\text { sociales. } \\
\text { Orbes y monitores de energía. }\end{array}$ \\
\hline Acción & $\begin{array}{l}\text { Reconocimiento social. } \\
\text { Programa de servicio social: Cuidado de } \\
\text { áreas verdes y forestación. }\end{array}$ \\
\hline
\end{tabular}

\begin{tabular}{|l|l|}
\hline & $\begin{array}{l}\text { Programa de servicio social: Limpieza de } \\
\text { paneles solares. } \\
\text { Orbes y monitores de energía. }\end{array}$ \\
\hline Mantenimiento & $\begin{array}{l}\text { Reconocimiento social. } \\
\text { Becas. } \\
\text { Opción de unirse a un equipo de mentores. } \\
\text { Sesiones de retroalimentación de buenas } \\
\text { prácticas del uso eficiente de la energía, } \\
\text { entre otros edificios y universidades. } \\
\text { Orbes y monitores de energía. }\end{array}$ \\
\hline
\end{tabular}

Tabla 4 Estrategias propuestas para diferentes etapas del cambio

Fuente: Elaboración Propia

De forma similar, en la Tabla 5, se enlistan las estrategias propuestas para cada segmento de la población descrito por la Teoría de la Difusión de las Innovaciones.

\begin{tabular}{|l|l|}
\hline Clasificación & \multicolumn{1}{|c|}{ Segmento } \\
\hline Innovadores & $\begin{array}{l}\text { Becas. } \\
\text { Evento de lanzamiento de la micro-red } \\
\text { virtual. } \\
\text { Formación temprana e incorporación } \\
\text { inicial de equipo de mentores/gestores. } \\
\text { Reconocimiento social - sitio web y } \\
\text { redes sociales. }\end{array}$ \\
\hline $\begin{array}{l}\text { Adoptadores } \\
\text { tempranos }\end{array}$ & $\begin{array}{l}\text { Evento de lanzamiento de la micro-red } \\
\text { virtual. } \\
\text { Participación en talleres de ahorro de } \\
\text { energía. } \\
\text { Reconocimiento social - sitio web y } \\
\text { redes sociales. } \\
\text { Participación en talleres de ahorro de } \\
\text { energía. }\end{array}$ \\
\hline $\begin{array}{l}\text { Manexión con mentores en etapas de } \\
\text { temprana } \\
\text { acción y mantenimiento - sitio web y } \\
\text { redes sociales. } \\
\text { Participación en talleres de ahorro de } \\
\text { energía. }\end{array}$ \\
\hline $\begin{array}{l}\text { Publicación de historias de éxito y de } \\
\text { integración comunitaria - sitio web y } \\
\text { redes sociales. }\end{array}$ \\
\hline tardía & $\begin{array}{l}\text { Apelar a normas sociales - redes } \\
\text { sociales. } \\
\text { Picnic. } \\
\text { Evento cultural. } \\
\text { Equipo/mobiliario para el edificio. } \\
\text { Intervenciones artísticas/estéticas para el } \\
\text { edificio. }\end{array}$ \\
\hline
\end{tabular}

Tabla 5 Estrategias propuestas para diferentes segmentos de la población en la adopción de innovaciones

Fuente: Elaboración Propia

La asignación de estrategias específicas por etapa y segmento de población no significa que carezcan de impacto para las demás categorías, únicamente representa que se consideran con mayor relevancia para el segmento que fueron asignadas. 


\section{Discusión}

Los resultados sugieren que métodos tecnológicos pueden ser vinculados de forma coherente con teorías de la psicología de la conducta, con el fin de diseñar estrategias para el ahorro de energía. Los resultados obtenidos del monitoreo energético, y su posterior aplicación en el diseño de una micro-red virtual, dan pauta a la integración de un marco conceptual que incorpora más de una modelo que describe el comportamiento humano, como lo sugieren Kollmuss y Agyeman (2002); los factores que definen el comportamiento proambiental son tan complejos que el analizarlos con únicamente un modelo o teoría no es suficiente. Por lo tanto, este trabajo vinculó el Modelo Transteórico, utilizado por He, Greenberg \& Huang (2010) para analizar el ahorro de energía mediante monitores de consumo, con la Teoría de la Difusión de las Innovaciones; una configuración que representa una novedad en el sector del ahorro energético.

El $67.4 \%$ y $65.4 \%$ de los encuestados declararon tener una percepción favorable sobre la inversión en equipo y mobiliario, y de un programa de becas, respectivamente, como estímulos dentro de un modelo de mercado energético. Mientras que el $71.7 \%$ consideraron la participación en talleres de ahorro de energía como una medida formativa pertinente, lo que resuena con las observaciones relacionadas a que el monitoreo en tiempo real no es suficiente para motivar la acción (Lynham et al., 2016; He, Greenberg \& Huang, 2010), y que la incorporación de políticas de ahorro energético orientadas al aprendizaje puede ser más efectiva que únicamente proyectar el consumo en tiempo real (Lynham et al., 2016).

Por esto, se consideraron distintas estrategias que fuesen más allá de este método. Particularmente, la propuesta de instalar orbes de consumo energético, con $75.5 \%$ de aceptación, mencionada por Timm \& Deal (2016), tuvo mayor aceptación frente a los monitores energéticos, con $69.2 \%$ de percepción positiva, sugiriendo que los primeros vuelven visible el consumo de energía de forma ligeramente más directa y sencilla.
Por otro lado, los resultados del monitoreo energético, presentados en la sección 3.1, revelan inconsistencias en la gestión del consumo de electricidad, que pueden ser tratadas mediante las estrategias propuestas. Por ejemplo, el Gráfico 3 muestra un consumo similar en periodos laborales y vacacionales en el Comedor, posiblemente debido la falta de estrategias para el ahorro de energía.

Los resultados de la sección anterior muestran diversas similitudes con la investigación realizada por Timm \& Deal (2016), donde se examinó el impacto de la información del consumo energético en tiempo real, en las actitudes y conductas de los ocupantes de un edificio. En su investigación, una de las conclusiones principales fue la importancia de considerar los distintos roles de los usuarios. Por esto, se decidió precisamente aplicar modelos que explican el cambio por etapas y segmentos de población, para la subsecuente asignación de estrategias por perfiles de usuarios. Sin embargo, será necesario evaluar la aceptación de estas con otros métodos, como entrevistas a profundidad.

Este trabajo presenta algunas limitaciones. Primero, el monitoreo energético tuvo algunos periodos de intermitencia, por lo que se tuvieron que excluir los datos de días que presentaron irregularidades en el funcionamiento de los sensores. Igualmente, se propone que en etapas futuras del proyecto se trabaje con un consumo meta variable, que se determine con base en los factores que modifican la generación y el consumo energético, como la irradiación y la temperatura ambiente.

Además, el periodo de monitoreo únicamente abarcó 8 meses, por lo que se sugiere volver a aplicar la metodología descrita al cumplir 1 año de monitoreo, en octubre de 2019, para así considerar las variaciones a través de la totalidad del ciclo escolar y de las estaciones. Por otro lado, fue hasta abril de 2019 que se contó con un funcionamiento estable en la totalidad de los inversores de la planta de generación, así que se propone que para un diseño más preciso de la micro-red se consideren los datos de generación de un año a partir de abril de 2019. 
Segundo, el comportamiento autoreportado puede diferir del comportamiento real. Para esto, se pueden utilizar otros métodos de recolección de datos, como monitoreo por video (Timm \& Deal, 2016), incrementando la veracidad de los datos de conducta. Tercero, es posible que los resultados de la encuesta estén sesgados, ya que la temática pudo haber sido particularmente llamativa para aquellas personas con afinidad a la conservación del medio ambiente o similares. Para esto, sería preferible realizar una aleatorización de la aplicación o procurar que la totalidad de los miembros de la comunidad universitaria contestaran la encuesta.

Finalmente, cabe añadir que posiblemente el mayor reto que enfrenta el cambio de conducta para el ahorro energético es la dificultad para consolidarlo (Asensio \& Delmas, 2016). Las campañas deben ser permanentes y estar en constante evaluación y rediseño. Éstas deben volverse intrínsecas a la forma en la que se interactúa con las edificaciones, para así generar un cambio de actitud en sus usuarios a largo plazo.

\section{Conclusiones}

Este artículo describe el diseño de una micro-red virtual fotovoltaica, aplicando monitoreo energético en tiempo real y estrategias para el cambio de la conducta; la integración de un estudio energético con modelos utilizados en las ciencias sociales. La incorporación particular del Modelo Transteórico con la Teoría de la Difusión de las Innovaciones ha sido un marco de trabajo novedoso para el campo del ahorro energético en edificaciones que forman parte de Centros Universitarios

En cuanto al monitoreo energético en tiempo real, se propone utilizar un porcentaje meta variable para el consumo de cada edificio en futuras etapas del proyecto, así como una reasignación de módulos, a partir de una base de datos con mayor resolución temporal de consumo y generación. Por otro lado, la conceptualización propia de las PGVs demostró ser conveniente al formular la propuesta de asignación de módulos fotovoltaicos, ya que brindó flexibilidad.
Además, durante el proceso de diseño, fue evidente el carácter multidisciplinario del proyecto, por lo que, será necesaria la integración de especialistas en las áreas que abarca esta investigación para su desarrollo óptimo. Futuras etapas de este proyecto deberán considerar estos aspectos.

Este trabajo busca plantear las bases de la implementación futura de un programa de Mercado Energético piloto en el Centro Universitario de Tonalá, junto con una intervención social, fundamentada en teorías del comportamiento humano. Los resultados aquí presentados servirán de antecedente para medir el impacto de las estrategias tomadas. Finalmente, cabe destacar que se contribuye a la creciente necesidad de integrar el desarrollo tecnológico con el estudio del comportamiento humano, al incorporar el concepto de microredes virtuales con teorías del cambio de la conducta.

\section{Agradecimientos}

Este trabajo fue apoyado por la convocatoria de SEP-CONACYT-Ciencia Básica bajo el proyecto 250777.

\section{Referencias}

Allouhi, A., El Fouih, Y., Kousksou, T., Jamil, A., Zeraouli, Y., \& Mourad, Y. (2015). Energy consumption and efficiency in buildings: current status and future trends. Journal of Cleaner production, 109, 118-130.

Alpizar-Castro, I., \& Rodríguez-Monroy, C. (2016). Review of Mexico's energy reform in 2013: Background, analysis of the reform and reactions. Renewable and Sustainable Energy Reviews, 58, 725-736.

Asensio, O. I., \& Delmas, M. A. (2016). The dynamics of behavior change: Evidence from energy conservation. Journal of Economic Behavior \& Organization, 126, 196-212.

Block, A (2 de diciembre de 2014). Glowing orbs illuminate Harrison and Rodin energy consumption. The Daily Pennsylvanian. Recuperado de: https://www.thedp.com/article/2014/12/pennorb -initiative-measures-energy-consumption 
Buchanan, K., Russo, R., \& Anderson, B. (2014). Feeding back about eco-feedback: How do consumers use and respond to energy monitors?. Energy Policy, 73, 138-146.

Burgess, J., \& Nye, M. (2008). Re-materialising energy use through transparent monitoring systems. Energy policy, 36(12), 4454-4459.

Geels, F. W., \& Schot, J. (2007). Typology of sociotechnical transition pathways. Research policy, 36(3), 399-417.

He, H. A., Greenberg, S., \& Huang, E. M. (2010, April). One size does not fit all: applying the transtheoretical model to energy feedback technology design. In Proceedings of the SIGCHI conference on human factors in computing systems (927-936). ACM.

Kaiser, F. G., Hübner, G., \& Bogner, F. X. (2005). Contrasting the Theory of Planned Behavior with the Value- Belief- Norm Model in Explaining Conservation Behavior. Journal of applied social psychology, 35(10), 2150-2170.

Kollmuss, A., \& Agyeman, J. (2002). Mind the gap: why do people act environmentally and what are the barriers to pro-environmental behavior?. Environmental education research, 8(3), 239-260.

Li, C., Hong, T., \& Yan, D. (2014). An insight into actual energy use and its drivers in highperformance buildings. Applied energy, 131, 394-410.

Lynham, J., Nitta, K., Saijo, T., \& Tarui, N. (2016). Why does real-time information reduce energy consumption?. Energy Economics, 54, 173-181.

McKenzie-Mohr, D. (2011). Fostering sustainable behavior: An introduction to community-based social marketing. Canadá: New Society Publishers.

Nutbeam, D., Harris, E., \& Wise, W. (2010). Theory in a nutshell: a practical guide to health promotion theories. Sídney, Australia: McGrawHill.
Operadora Universitaria de Energía (2017). Colaboración con el Programa Integral de Transición Energética. Guadalajara, México: Universidad de Guadalajara. Recuperado de: http://www.energiauniversitaria.com/

Petersen, J. E., Shunturov, V., Janda, K., Platt, G., \& Weinberger, K. (2007). Dormitory residents reduce electricity consumption when exposed to real-time visual feedback and incentives. International Journal of Sustainability in Higher Education, 8(1), 16-33.

Saboori, H., Mohammadi, M., \& Taghe, R. (2011, March). Virtual power plant (VPP), definition, concept, components and types. In 2011 Asia-Pacific Power and Energy Engineering Conference (pp. 1-4). IEEE.

Sovacool, B. K. (2014). Diversity: Energy studies need social science. Nature, 511(7511), 529.

Stern, P. C., Sovacool, B. K., \& Dietz, T. (2016). Towards a science of climate and energy choices. Nature Climate Change, 6(6), 547.

Sun, K., Yan, D., Hong, T., \& Guo, S. (2014). Stochastic modeling of overtime occupancy and its application in building energy simulation and calibration. Building and Environment, 79, 1-12.

Timm, S. N., \& Deal, B. M. (2016). Effective or ephemeral? The role of energy information dashboards in changing occupant energy behaviors. Energy Research \& Social Science, 19, 11-20.

Völlink, T., Meertens, R. E. E., \& Midden, C. J. (2002). Innovating 'diffusion of innovation' theory: innovation characteristics and the intention of utility companies to adopt energy conservation interventions. Journal of environmental psychology, 22(4), 333-344. 